\title{
Identification of Isomorphism and Distinct Mechanisms Using Square Adjacency Matrix
}

\author{
Sayeed Ahamad ${ }^{1}$, Aas Mohammad ${ }^{2}$ \\ ${ }^{1}$ M.Tech Student, Department of Mechanical Engineering, Jamila Millia Islamia University, New Delhi, India \\ ${ }^{2}$ Professor, Department of Mechanical Engineering, Jamila Millia Islamia University, New Delhi, India
}

\begin{abstract}
For obtaining the structurally distinct mechanisms of the kinematic chains, link disposition matrix, flow matrix and row matrix of adjacency matrix are used. These methods shows either a lack of uniqueness or it consumes a lot of time. Determination of distinct mechanism using flow matrix is very long process. Therefore to overcome this problem we are trying to develop a computationally efficient method for generalizing the planer kinematic chains having same number of links but different kinematic pairs to determining the distinct mechanisms from the given kinematic chains which is called Squared Adjacency Matrix [SAM]. In the present work the graph theory is applied in the modelling of kinematic chains and mechanisms. A new matrix is obtained from the kinematic graph of the kinematic chains, called "Squared Adjacency Matrix". Fixing the links of 1 - links kinematic chain, 1 mechanisms are generated. Similarly other links are fixed and other mechanisms are obtained. Some of them are equivalent and others are different. If the corresponding row and column entries of the square adjacency matrix are changed to $a_{11}=1$ and remaining elements are zero as turn in turn, $n$-square adjacency matrices are obtained and it is representation of $n$-mechanisms. Therefore the new structural invariants are obtained from $n$-square adjacency matrices. These invariants are identical for structurally equivalent mechanisms and distinct for distinct mechanisms. Therefore number of distinct mechanisms can be obtained from the given kinematic chain. The proposed method is applied to determine the distinct mechanisms of single degree of freedom, six - links, eight - links and ten links kinematic chains
\end{abstract}

Keywords: Kinematic chains; Structural Invariants; Isomorphism; Squared Adjacency Matrix; Determinants

\section{Introduction}

In a mechanism design problem, the systematic steps are type synthesis, structural/number synthesis, and dimensional synthesis. Structural synthesis of the kinematic chain and mechanism has been the subject of a number of studies in recent years. One important aspect of structural synthesis is to develop all possible arrangements of kinematic chains and also their derived mechanisms for a given number of links, joints, and degree of freedom, so that the designer has the liberty to select the best or optimum mechanism according to his requirements. In the course of development of kinematic chains and mechanisms, duplication may be possible. For this reason, many methods have been proposed by researchers to identify duplication or, in other words, to detect isomorphism among the kinematic chains. Most of these methods are based on the adjacency matrix [3] and the distance matrix [4]. To determine the structurally distinct mechanisms of a kinematic chain, the link disposition method [5], the flow matrix method [6], and the row sum of extended distance matrix methods [7] are used. Minimum code [8], characteristic polynomial of matrix [9], identification code [10], link path code [11], summation polynomial [12], etc. are used to characterize the kinematic chains. These methods, however, either lack uniqueness or they take too much time. Determination of all distinct mechanisms from an n-link kinematic chain using flow matrix [6] is a very lengthy process because n-flow matrices are required. Hence, there is a need to develop a computationally efficient method to determine the distinct mechanisms of a kinematic chain. In the present work, a new method is proposed to determine the distinct mechanisms of a kinematic chain. The structural invariants i.e. determinants values are the same for identical or structurally equivalent mechanisms and different for distinct mechanisms. So, it is possible to identify all distinct mechanisms derived from given kinematic chains. The invariants may also be used to characterize the mechanisms and to detect isomorphism in kinematic chains. The new method is explained with the help of examples of planer kinematic chains having all simple joints or a combination of simple and multiple joints. The results of this paper are useful for identifying distinct mechanisms efficiently and will help designers to select the best possible mechanism for a required task in the initial stage of design.

\section{Graph of the Kinematic Chain}

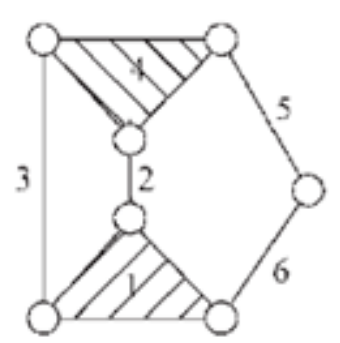

(a) Stephenson Chain (b) Kinematic Graph

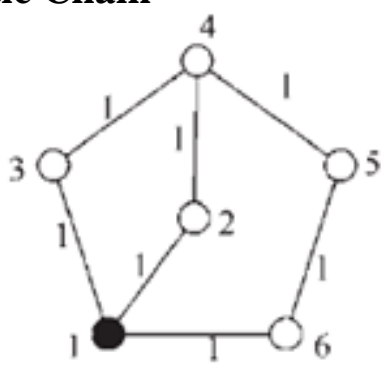

(All Simple Joints)

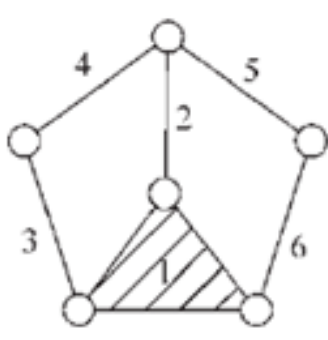

(c) Kinematic Chain

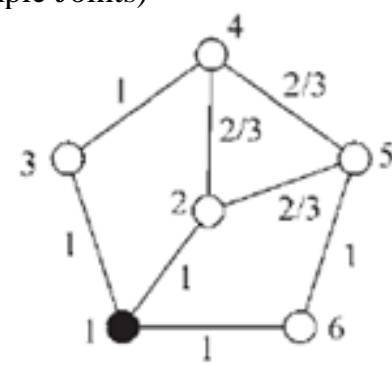

(d) Kinematic Graph
(One Double Joint)

Figure 1: 6 - links, 7 - joints, $1-F$, Kinematic Chains and their Kinematic Graphs 


\section{International Journal of Science and Research (IJSR) \\ ISSN (Online): 2319-7064}

Index Copernicus Value (2013): 6.14 | Impact Factor (2015): 6.391

Let us represent a kinematic chain with the help of a graph (it is also called the kinematic graph). Let vertices of the kinematic graph represent the links numbered 1, 2, 3, 4, 5, 6 and edges represent the joints of the kinematic chain. There are two types of kinematic chains: one having all simple joints like Stephenson's chain and another having simple and/or multiple joints as shown in Figure 4.1 (a) and 4.1 (c) respectively. The number of edges in the kinematic graph is generally equal to the number of joints in the case of simple jointed kinematic chains. But in the case multiple jointed kinematic chains, the number of edges is more as compare to the number of joints as shown in Figure 4.1 (d). To avoid this controversy, a joint value is assigned to each of the joint connecting $i^{\text {th }}$ link to $j^{\text {th }}$ link of a kinematic chain. The value will be given as:

$$
\mathbf{J}_{\mathrm{v}}=2 /\left(\mathbf{J}_{\mathrm{k}}+\mathbf{1}\right)
$$

Where: $\mathrm{J}_{\mathrm{v}}=$ Joint value, and

$\mathrm{J}_{\mathrm{k}}=$ Type of the joint

For simple joint, $\mathbf{J}_{\mathrm{k}}=1, \mathrm{~J}_{\mathrm{v}}=1$

For double joint, $\mathrm{J}_{\mathrm{k}}=2, \mathrm{~J}_{\mathrm{v}}=2 / 3$

For triple joint, $\mathrm{J}_{\mathrm{k}}=3, \mathrm{~J}_{\mathrm{v}}=2 / 4=1 / 2$

For quaternary joint, $\mathrm{J}_{\mathrm{k}}=4, \mathrm{~J}_{\mathrm{v}}=2 / 5$

For $\mathrm{n}$ - tuple joint, $\mathrm{J}_{\mathrm{k}}=\mathrm{n}, \mathrm{J}_{\mathrm{v}}=2 /(\mathrm{n}+1)$

Therefore, each edge of the kinematic graph connecting $i^{\text {th }}$ vertex to $\mathrm{j}^{\text {th }}$ vertex is assigned with the corresponding joint value. For example the joint value of each edge is 1 in the kinematic graph as shown in Figure 4.1 (b). The joint value of each edges joining vertices $13,34,16,12$, and 56 is 1 , whereas it is $2 / 3$ for the edge joining the vertices 24,45 , and 25 in Figure 4.1 (d). The sum of the all joint values is 7 , i.e. $(5 \times 1+3 \times 2 / 3)$ which is actually related to the number of simple joints existing in a kinematic chain.

\section{Matrix Representation of the Kinematic Graph of A Kinematic Chain}

The $(0,1)$ adjacency matrix and the distance matrix are generally used to represent the kinematic graph of a kinematic chain. The adjacency matrix shows only the connectivity between adjacent vertices/links. The distance matrix has also the relation between the links that are not directly connected to each other in the form of shortest path distance. But both adjacency and distance matrices are not able to furnish the information about the types of links those are directly connected with a joint or with the shortest path distances respectively. These matrices are also not able to distinguish the type of the joints in the multiple - jointed kinematic chains. A generalized matrix representation is made in the literature [T.S. Mruthyunjaya and M.R. Raghavan, 1979] in which the elements of the adjacency matrix $a_{i j}$ represents the multiplicity (type) of the joint. The value of the $a_{i j}$ is 1 if the joint between $i^{\text {th }}$ and $j^{\text {th }}$ link is a simple joint, 2 if it is double joint, 3 if it is ternary joint, 4 , if it is quaternary joint and so on. In the present work, there is a slight change in the methodology.

\subsection{Square Adjacency Matrix [Sam]}

$[\mathrm{SAM}]$ matrix representation may be given as:

Where:

$$
[\mathbf{S A M}]=\left\{\mathbf{P}_{\mathrm{ij}}\right\}
$$

$\mathrm{P}_{\mathrm{ij}}=$ Square of the sum of the joint value in [SAM] between $\mathrm{i}^{\text {th }}$ and $\mathrm{j}^{\text {th }}$ link those are connected directly (i.e., $\mathrm{i}$ is not equal to $\mathrm{j}$ ).

Otherwise: $P_{i j}=0$ i.e. those are not connected directly.

$P_{i i}=$ Square of the degree of the $i^{\text {th }} \operatorname{link}(i=j)$

Off course: $\mathrm{P}_{\mathrm{ij}}=0$

\subsubsection{Representation of Square Adjacency Matrix [SAM]} The Square Adjacency Matrix [SAM] matrix is represented as:

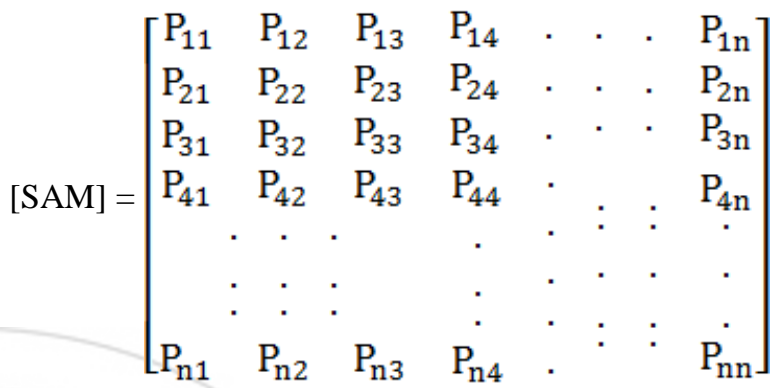

3.1.2. Structural Invariants for Kinematic Chains

The structural invariant is the determinant value of the [SAM] matrix. These determinants value of the [SAM] that is invariants are able to detect the isomorphism among the kinematic chains having simple joints, multiple joints. These invariants are unique and used as identification numbers to detect the isomorphism between the simple and multiple jointed kinematic chains.

\subsubsection{Isomorphism of the Kinematic Chains}

\section{Theorem:}

"Similar matrices have the same determinant values". It means that if the determinant values of the two [SAM] matrices representing two kinematic chains are same, their structural invariants will be same and the two kinematic chains are treated as isomorphic chains.

\section{Distinct Mechanisms of A Kinematic Chain}

The basis of a mechanism is the kinematic chain. With the help of inversions, each chain leads to as many mechanisms as there are links in the chain. Some of the mechanisms/inversions are equivalent and therefore should be counted as one. For this reason, no appropriate mathematical equation has yet been found which tells how many different types of mechanisms, each having same number of links and joints, can be derived from a given kinematic chain. Therefore, it is needed to develop an efficient algebraic method to identify the number of distinct mechanisms for a given family of kinematic chains so that designer has a choice to select the best suited mechanism to fulfil their requirements.

\subsection{Identification of Structurally Equivalent Links and Distinct Mechanisms}

In chapter -4 , a kinematic chain is modelled by its kinematic graph and then represented by the [SAM] matrix. When any link of a kinematic chain is fixed, a mechanism is obtained. If in the $[S A M]$ matrix the elements of the $i^{\text {th }}$ row and $i^{\text {th }}$ 


\section{International Journal of Science and Research (IJSR) \\ ISSN (Online): 2319-7064}

Index Copernicus Value (2013): 6.14 | Impact Factor (2015): 6.391

column are changed to $1\left(\mathrm{P}_{\mathrm{ii}}=1\right)$, and remaining all to zero, it will be a representation of the mechanism with $i^{\text {th }}$ link fixed. Then this new matrix is represented by [SAM i] matrix. (where, $\mathrm{i}=1,2,3,4 \ldots \ldots \ldots \ldots$ ). For example, to represent the first mechanism the elements of the first row and column of [SAM] matrix are changed to zero, but $\mathrm{P}_{11}=1$ and this new matrix is designated as [SAM 1] matrix.

The determinant value of the [SAM i] matrix is called structural invariant. This structural invariant is the characteristic number of the $\mathrm{i}^{\text {th }}$ mechanism. This process is repeated for $\mathrm{i}=1$ to $\mathrm{n}$. In this way a set of $\mathrm{n}-$ structural invariants are obtained, some of them may be same and others are different. The same structural invariants represent the corresponding structurally equivalent links constitute 1 distinct mechanism.

\subsection{Structural Invariant of the Kinematic Chain}

The structural invariant from [SAM] matrix using software MATLAB for the kinematic chain shown in figure 1 (a) is: $\operatorname{det}\left(\mathrm{A}_{1}\right)=16224$

The structural invariant that is the determinant values is used as the identification number of the kinematic chain shown in figure 1 (a).

\subsection{Matrix Representation of the Mechanisms}

Fixing the link -1 , the first mechanism is developed. Hence, changing the elements of first row and first column zero and $\mathrm{P}_{11}=1$, of the [SAM] matrix, the [SAM 1] matrix is obtained and shown as:

In $\mathrm{n}$ - link mechanisms, let us assume that

$\mathrm{d}=$ Number of distinct links having distinct invariants.

$\mathrm{e}=$ Number of sets equivalent links.

$\mathrm{t}=$ Total number of distinct mechanisms. $\mathbf{t}=\mathbf{d}+\mathbf{e}$

The form of the [SJVM 1] matrix representing the mechanism with link -1 fixed will be:

$$
\text { SJVM 1 }=\left[\begin{array}{cccccccccc}
1 & 0 & 0 & 0 & 0 & 0 & 0 & & 0 \\
0 & P_{22} & P_{23} & P_{24} & P_{25} & P_{26} & * & P_{2 n} \\
0 & P_{32} & P_{33} & P_{34} & P_{35} & P_{36} & * & P_{3 n} \\
0 & P_{42} & P_{43} & P_{44} & P_{45} & P_{46} & & P_{4 n} \\
0 & P_{52} & P_{53} & P_{54} & P_{55} & P_{56} & : & P_{5 n} \\
0 & P_{62} & P_{63} & P_{64} & P_{65} & P_{66} & * & P_{n 8} \\
& 0 & * & * & * & * & : & * \\
0 & P_{n 2} & P_{n 3} & P_{n 4} & P_{n 5} & P_{n 6} & & P_{n n}
\end{array}\right]
$$

\subsection{Structural Invariants of the First Mechanism}

The structural invariants that is determinant values of the first mechanism is derived from the [SAM 1] matrix of the kinematic chain [Figure 1 (a)] is given as:

$\operatorname{det}\left(\mathrm{A}_{11}\right)=1976$

Similarly, changing the row and column elements to zero, but $\mathrm{P}_{\mathrm{ii}}=1$ of the $[\mathrm{SAM}]$ matrix turn in turn, structural invariants of the other mechanisms are obtained and given as:

$\operatorname{det}\left(A_{12}\right)=4316$

$\operatorname{det}\left(\mathrm{A}_{13}\right)=4316$

$\operatorname{det}\left(\mathrm{A}_{14}\right)=1976$

$\operatorname{det}\left(\mathrm{A}_{15}\right)=4472$

$\operatorname{det}\left(\mathrm{A}_{16}\right)=4472$

To identify the distinct mechanisms derived from a planer kinematic chain, let us consider that a configuration of 6 links, 7 - joints, 1 - F Stephenson's chain and its kinematic graph as shown in the figure -1 (a) and 1 (b) respectively. The all joints of the Stephenson's chain are simple. Therefore, each edge of the kinematic graph connecting $i^{\text {th }}$ vertex to $\mathrm{j}^{\text {th }}$ vertex is assigned with the joint value 1 .

\subsection{Square Adjacency Matrix}

The Square Adjacency Matrix representing the kinematic graph (Fig. 5.1 b) using equation 4.3 is written as:

$$
[\mathrm{SAM}]=\left[\mathrm{A}_{1}\right]=\left[\begin{array}{llllll}
9 & 1 & 1 & 0 & 0 & 1 \\
1 & 4 & 0 & 1 & 0 & 0 \\
1 & 0 & 4 & 1 & 0 & 0 \\
0 & 1 & 1 & 9 & 1 & 0 \\
0 & 0 & 0 & 1 & 4 & 1 \\
1 & 0 & 0 & 0 & 1 & 4
\end{array}\right]
$$

\subsection{Identification of the Distinct Mechanisms}

Observing the structural invariants that is determinant values for the above 6 - mechanisms, it is found that the structural invariants of the link -1 and link -4 are the same, hence they are treated as structurally equivalent links and they form only one distinct mechanism. Similarly invariants of the link -2 , link - 3 are same, hence they form second distinct mechanism and the invariants of link -5 and link -6 are also same, hence they form third distinct mechanism. So, we can write:

$\mathrm{e}=3$ (Number of set of equivalent links)

$\mathrm{d}=0$ (Number of distinct links)

$\mathrm{t}=\mathrm{d}+\mathrm{e}$

$\mathrm{t}=3+0=3$

Therefore, 3 distinct mechanisms are obtained from Stephenson's kinematic chain as shown in figure 1 (a). 


\section{International Journal of Science and Research (IJSR) \\ ISSN (Online): 2319-7064}

Index Copernicus Value (2013): 6.14 | Impact Factor (2015): 6.391

\section{Illustrative Example - 2 (Multiple Jointed Kinematic Chain)}

Let us take an example of a 6-link 7-joint (5 simple joints and 1 double joint) 1-F kinematic chain and its kinematic graph, shown in Figs 1(c) and (d), respectively. The joint value of the edges joining the vertices $13,16,56,34$, and 12 is 1 and that the edges joining the vertices 24,25 , and 45 is $2 / 3$.

\subsection{Square Joint Value Matrix}

The Square Adjacency Matrix representing the kinematic graph (Fig. $5.1 \mathrm{~d}$ ) using equation 4.3 is written as:

$\left[\mathrm{A}_{2}\right]=\left[\begin{array}{llllll}9.00 & 1.00 & 1.00 & 0.00 & 0.00 & 1.00 \\ 1.00 & 4.00 & 0.00 & 0.44 & 0.44 & 0.00 \\ 1.00 & 0.00 & 4.00 & 1.00 & 0.00 & 0.00 \\ 0.00 & 0.44 & 1.00 & 4.00 & 0.44 & 0.00 \\ 0.00 & 0.44 & 0.00 & 0.00 & 4.00 & 1.00 \\ 1.00 & 0.00 & 0.00 & 0.00 & 1.00 & 4.00\end{array}\right]$

\subsection{Structural Invariant of the Kinematic Chain}

The structural invariant from [SAM] matrix using software MATLAB for the kinematic chain shown in figure 1 (c) is: $\operatorname{det}\left(\mathrm{A}_{2}\right)=7093.2$

The structural invariant that is the determinant values is used as the identification number of the kinematic chain shown in figure 1 (c).

\subsection{Structural Invariants of the Mechanisms}

Fixing the link -1 , the first mechanism is developed. Hence, changing the elements of first row and first column zero and $\mathrm{P}_{11}=1$, of the $[\mathrm{SAM}]$ matrix, the [SAM 1] matrix is obtained and shown as:

$\left[\mathrm{A}_{21}\right]=\left[\begin{array}{llllll}1.00 & 0.00 & 0.00 & 0.00 & 0.00 & 0.00 \\ 0.00 & 4.00 & 0.00 & 0.44 & 0.44 & 0.00 \\ 0.00 & 0.00 & 4.00 & 1.00 & 0.00 & 0.00 \\ 0.00 & 0.44 & 1.00 & 4.00 & 0.44 & 0.00 \\ 0.00 & 0.44 & 0.00 & 0.44 & 4.00 & 1.00 \\ 0.00 & 0.00 & 0.00 & 0.00 & 1.00 & 4.00\end{array}\right]$

$\operatorname{det}\left(\mathrm{A}_{21}\right)=866.4636$

Similarly, changing the row and column elements to zero and $\mathrm{P}_{\mathrm{ii}}=1$, of the [SAM] matrix turn in turn, structural invariants of the other mechanisms are obtained and given as:

$\operatorname{det}\left(\mathrm{A}_{22}\right)=1879$

$\operatorname{det}\left(\mathrm{A}_{23}\right)=1958.6$

$\operatorname{det}\left(\mathrm{A}_{24}\right)=1945.6$

$\operatorname{det}\left(\mathrm{A}_{25}\right)=1945.6$

$\operatorname{det}\left(\mathrm{A}_{26}\right)=1958.6$

\subsection{Identification of the Distinct Mechanisms}

Observing the structural invariants that is determinant values for the above 6 -mechanisms, it is found that the structural invariants of the link -3 and link -6 are the same, hence they are treated as structurally equivalent links and they form only one distinct mechanism. Similarly invariants of the link -4 link -5 , are same and they form second distinct mechanisms. So, we can write:

$\mathrm{e}=2$ (Number of set of equivalent links)

$\mathrm{d}=2$ (Number of distinct links)

$\mathrm{t}=\mathrm{d}+\mathrm{e}$

$\mathrm{t}=2+2=4$

Therefore, 4 distinct mechanisms are obtained from multiple jointed kinematic chain as shown in figure 1 (c).

\section{Illustrative Example - 3 (Isomorphism among the Kinematic Chains having Co-spectral Graphs)}

Graphs having the same determinant value are called as CoSpectral graphs. These determinant values are derived from the $[\mathrm{SAM}]$ matrix, but their determinant values that is invariants derived from $[\mathrm{SAM}]$ matrices are distinct. This will be proved with the help of the examples of 10-link, 12joint," 3-F kinematic chains shown in Figs 2 (a) and (c). Figures 2 (b) and (d) represent the kinematic graphs of these kinematic chains, respectively.
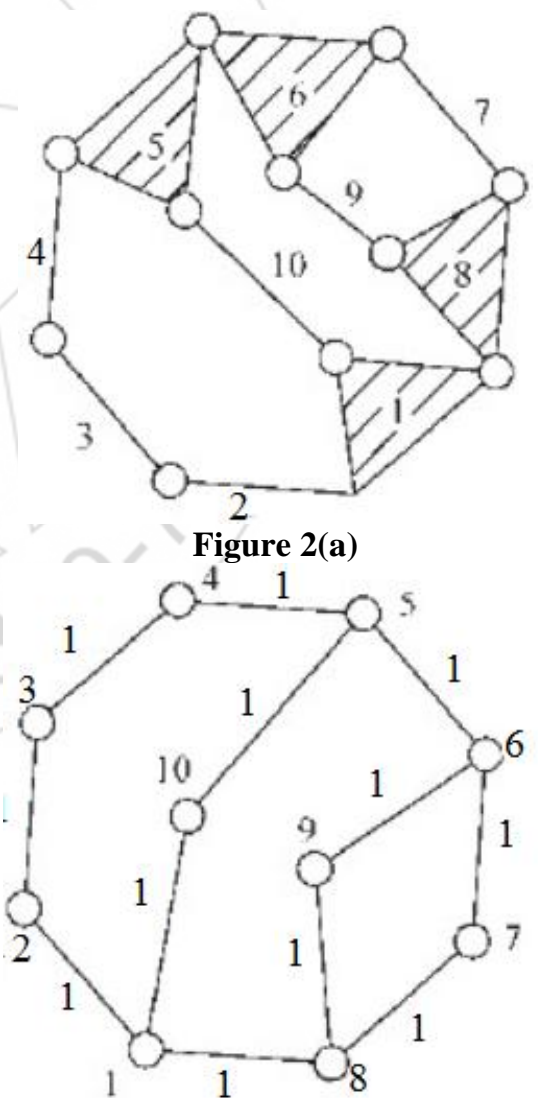

Figure 2(b) 


\section{International Journal of Science and Research (IJSR) \\ ISSN (Online): 2319-7064}

Index Copernicus Value (2013): 6.14 | Impact Factor (2015): 6.391

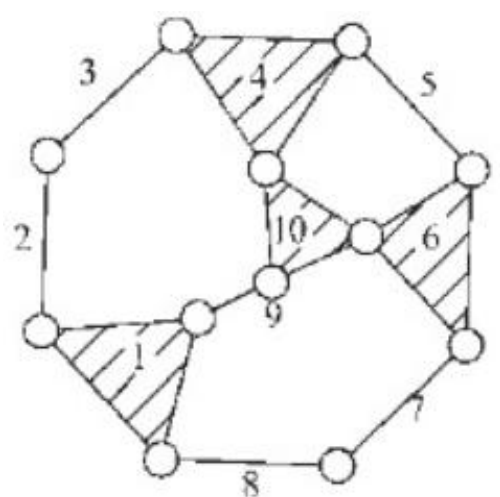

Figure 2(c)

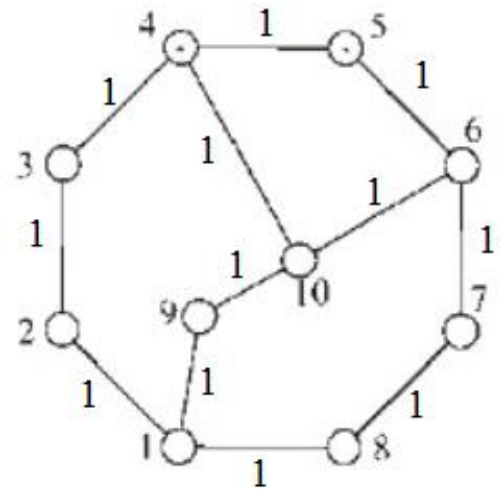

Figure 2(d)

Figure 2: 10 - Links, 12 - Joints, 3 - F Kinematic Chains and their Co-Spectral Graphs

\subsection{Structural Invariants of the Co-Spectral Graphs}

Following the same procedure, the structural invariants of the kinematic graphs shown in Figures. 2 (b) and 2 (d), derived from the Square Adjacency Matrix, are written as:

The Square Adjacency Matrix representing the kinematic graph (Fig. 2 b) using equation (2) is written as:

$[\mathrm{SAM}]=\left[\mathrm{A}_{3}\right]=\left[\begin{array}{llllllllll}9 & 1 & 0 & 0 & 0 & 0 & 0 & 1 & 0 & 1 \\ 1 & 4 & 1 & 0 & 0 & 0 & 0 & 0 & 0 & 0 \\ 0 & 1 & 4 & 1 & 0 & 0 & 0 & 0 & 0 & 0 \\ 0 & 0 & 1 & 4 & 1 & 0 & 0 & 0 & 0 & 0 \\ 0 & 0 & 0 & 1 & 9 & 1 & 0 & 0 & 0 & 1 \\ 0 & 0 & 0 & 0 & 1 & 9 & 1 & 0 & 1 & 0 \\ 0 & 0 & 0 & 0 & 0 & 1 & 4 & 1 & 0 & 0 \\ 1 & 0 & 0 & 0 & 0 & 0 & 1 & 9 & 1 & 0 \\ 0 & 0 & 0 & 0 & 0 & 1 & 0 & 1 & 4 & 0 \\ 1 & 0 & 0 & 0 & 1 & 0 & 0 & 0 & 0 & 4\end{array}\right]$

The structural invariant from [SAM] matrix using software MATLAB for the kinematic chain shown in figure 2 (a) is: $\operatorname{det}\left(\mathrm{A}_{3}\right)=18083000$

The structural invariant is used as the identification number of the kinematic chain shown in figure 2 (a).

The Square Adjacency Matrix representing the kinematic graph (Fig. 2 d) using equation (2) is written as:

$$
\left[\mathrm{A}_{4}\right]=\left[\begin{array}{llllllllll}
9 & 1 & 0 & 0 & 0 & 0 & 0 & 1 & 1 & 0 \\
1 & 4 & 1 & 0 & 0 & 0 & 0 & 0 & 0 & 0 \\
0 & 1 & 4 & 1 & 0 & 0 & 0 & 0 & 0 & 0 \\
0 & 0 & 1 & 9 & 1 & 0 & 0 & 0 & 0 & 1 \\
0 & 0 & 0 & 1 & 4 & 1 & 0 & 0 & 0 & 0 \\
0 & 0 & 0 & 0 & 1 & 9 & 1 & 0 & 0 & 1 \\
0 & 0 & 0 & 0 & 0 & 1 & 4 & 1 & 0 & 0 \\
1 & 0 & 0 & 0 & 0 & 0 & 1 & 4 & 0 & 0 \\
1 & 0 & 0 & 0 & 0 & 0 & 0 & 0 & 4 & 1 \\
0 & 0 & 0 & 1 & 0 & 1 & 0 & 0 & 1 & 9
\end{array}\right]
$$

\subsection{Structural Invariant of the Kinematic Chain}

The structural invariant from [SAM] matrix using software MATLAB for the kinematic chain shown in fig 2 (c) is: $\operatorname{det}\left(\mathrm{A}_{4}\right)=18073284$

The structural invariant is used as the identification number of the kinematic chain shown in figure 2 (c).

The structural invariants of the above two Co-Spectral graphs shown in figure 2 (b) and 2(d) are different, therefore both the graphs and in turn the kinematic chains shown in figure 2 (a) and 2 (c) are non - isomorphic.

\section{Results}

The proposed invariants i.e. determinants values are used as the identification number of the kinematic chains having simple joints. These invariants are also able to detect the isomorphism among the kinematic chains having simple joints, multiple joints

\section{Conclusions}

- From the proposed graph of the kinematic chain, a new matrix is derived and which is called square joint value matrix [SAM].

- $[\mathrm{SAM}]$ is able to identify the type of the joints existing in a kinematic chain.

- These determinants value of the [SAM] that is invariants are able to detect the isomorphism among the kinematic chains having simple joints, multiple joints.

- Using the proposed method the number of distinct mechanisms derived from the $1-\mathrm{F}, 8$ - links kinematic chains are 72 the proposed method can successfully be used to detect the isomorphism in the kinematic chains having $10-$ links.

\section{References}

[1] Sayeed Ahamad, Aas Mohammad and Jiaul Mustafa, "Identification of Isomorphism of Kinematic Chains using different types of Kinematic Pairs", International Journal of Engineering Sciences \& Research Technology (IJESRT), Vol 5 Issue 3, pp 678-683, March 2016.

[2] Sayeed Ahamad and Aas Mohammad, "Generalized Representataion of Planer Kinematic Chains having different types of Kinematic Pairs”, International Journal 


\section{International Journal of Science and Research (IJSR) \\ ISSN (Online): 2319-7064 \\ Index Copernicus Value (2013): 6.14 | Impact Factor (2015): 6.391}

of Computational Engineering Management (IJCEM), Vol 19 Issue 3,May 2016.

[3] Uicker, J. J. and Raicu, A. A method for the identification and recognition of equivalence of kinematic chains. Mech. Mach. Theory, 1975, 10, 375383.

[4] Rao, A. C. Kinematic chains, isomorphism, inversions and type of freedom, using the concept of Hamming distances. Indian J. Tech., 1988, 26, 105-109.

[5] Mruthyunjaya, T. S. and Raghavan, M. R. Computer aided analysis of the structural synthesis of kinematic chains. Mech. Mach. Theory, 1984, 19, 357-368.

[6] Nageswara Rao, C. and Rao, A. C. Selection of best frame, input and output links for function generators modeled as probabilistic system. Mech. Mach. Theory, 1996, 31, 973-983.

[7] Mohammad, A. and Agrawal, V. P. Identificationand isomorphism of kinematic chains and mechanisms. $11^{\text {th }}$ ISME Conference, IIT Delhi, India, 1999, pp. 197-202.

[8] Ambekar, A. G. and Agrawal, V. P. Identification of kinematic generator using min. codes. Mech. Mach. Theory, 1987, 22(5), 463-471.

[9] Mruthyunjaya, T. S. and Raghavan, M. R. Structural analysis of kinematic chains and mechanisms basedon matrix representation. Trans. ASME, J. Mech. Des., 1979, 101, 488-518.

[10] Ambekar, A. G. and Agrawal, V. P. Identification and classification of kinematic chains and mechanisms using identification codes. The Fourth International Symposium on Linkage and computer aided design methods, Bucharest, Romania, 1985, Vol. 1(3), pp. 545 552.

[11] Agrawal, V. P., Yadav, J. N., and Pratap, C. R. Mechanism of kinematic chain and the degree of structural similarity based on the concept of link - path code. Mech. Mach. Theory, 1996, 31(7), 865-871.

[12] Shende, S. and Rao, A. C. Isomorphism in kinematic chains. Mech. Mach. Theory, 1994, 29(77), 1065-1070.

[13]Zichos, H. Tribology - a system approach to science and technology of friction, lubrication and wear, tribologyseries-1, 1978 (Elsevier, Amesterdam).

[14] Czichos, H. System approach to the analysis of wear problems. Conference of Lubrication, Friction and Wear in Engineering, Melbourne, Australia, 1980, pp. 247252.

[15] Gandhi, O. P. and Agrawal, V. P. A diagraph approach to system wear evaluation and analysis. STLE/ASME Tribology Conference, New Oreleans, 1994, Vol. 116, pp. 268-274.

[16]Hsiung, C. Y. and Mao, G. Y. Linear algebra, 1998 (World Scientific Publishing, Singapore).

[17][Sayeed Ahamad, 2016] Sayeed Ahamad, Aas Mohammad and Jiaul Mustafa, "Identification of Isomorphism of Kinematic Chains using different types of Kinematic Pairs", International Journal of Engineering Sciences \& Research Technology (IJESRT), Vol 5 Issue 3, pp 678-683, March 2016.

[18][Sayeed Ahamad, 2016] Sayeed Ahamad and Aas Mohammad, "Generalized Representataion of Planer Kinematic Chains having different types of Kinematic Pairs", International Journal of Computational
Engineering Management (IJCEM), Vol 19 Issue 3, May 2016.

[19][M. Plecnik, 2015] The Kinematic Design of Six-bar Linkages Using Polynomial Homoyopy Continuation, Doctoral, University of Calfornia, Irvin, 2015.

\section{Author Profile}

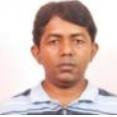

Sayeed Ahamad is M.Tech student, received B.Tech in Mechanical and Industrial Engineering from UPTU Lucknow, UP, and presently pursuing M.Tech in Machine Design from Jamia Millia Islamia University,New Delhi (India).

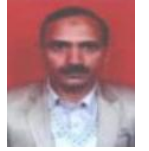

Professor Aas Mohammad is in Department of Mechanical Engineering, Jamia Millia Islamia. He is actively involved in research and Teaching and research field includes Theory of Machine, and Graph Theory. He has number of papers published in conference proceedings and journals. 